\title{
A Case of Young-Adult Stroke with Hyperhomocysteinemia and Orbitofrontal Syndrome
}

\author{
Deepak Suraparaju ${ }^{1} \&$ Mithun S. ${ }^{2}$ \\ ${ }^{1}$ DNB Neurosurgery, FRCS Neurosurgery Edinburgh, FEBNS Europe \\ Consultant Neurosurgeon, Department of Surgery, Srinivas Institute of Medical Sciences, \\ Mangalore, India \\ ${ }^{2}$ MD, DNB Psychiatry, Assistant Professor, Department of Psychiatry, Srinivas Institute of \\ Medical Sciences, Mangalore, India \\ E-mail: $\underline{\text { mdmitsmj@gmail.com }}$
}

Area/Section: Medical Sciences.

Type of the Paper: Medical Case Report.

Type of Review: Peer Reviewed as per $|\mathrm{C}| \mathrm{O}|\mathrm{P}| \mathrm{E} \mid$ guidance.

Indexed in: OpenAIRE.

DOI: http://doi.org/10.5281/zenodo.3997358

Google Scholar Citation: IJHSP

\section{How to Cite this Paper:}

Suraparaju, Deepak \& S., Mithun. (2020). A Case of Young-Adult Stroke with Hyperhomocysteinemia and Orbitofrontal Syndrome. International Journal of Health Sciences and Pharmacy (IJHSP), 4(2), 1-4. DOI: http://doi.org/10.5281/zenodo.3997358

International Journal of Health Sciences and Pharmacy (IJHSP)

A Refereed International Journal of Srinivas University, India.

(C) With Authors.

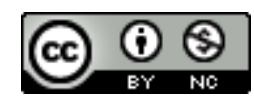

This work is licensed under a Creative Commons Attribution Non-Commercial 4.0 International License subject to proper citation to the publication source of the work.

Disclaimer: The scholarly papers as reviewed and published by the Srinivas Publications (S.P.), India are the views and opinions of their respective authors and are not the views or opinions of the SP. The SP disclaims of any harm or loss caused due to the published content to any party. 


\title{
A Case of Young-Adult Stroke with Hyperhomocysteinemia and Orbitofrontal Syndrome
}

\author{
Deepak Suraparaju ${ }^{1} \&$ Mithun S. $^{2}$ \\ ${ }^{1}$ DNB Neurosurgery, FRCS Neurosurgery Edinburgh, FEBNS Europe \\ Consultant Neurosurgeon, Department of Surgery, Srinivas Institute of Medical Sciences, \\ Mangalore, India \\ ${ }^{2}$ MD, DNB Psychiatry, Assistant Professor, Department of Psychiatry, Srinivas Institute of \\ Medical Sciences, Mangalore, India \\ E-mail: mdmitsmj@gmail.com
}

\begin{abstract}
Stroke in young can have a significant impact on survival of patients. Early diagnosis and intervention by a holistic team approach are vital in its management. Here we describe a case of stroke in a young 37 old male patient presenting with an uncommon cause of Hyperhomocysteinemia with subsequent post-stroke psychiatric manifestations. A holistic team approach model and challenges in management are discussed.
\end{abstract}

Keywords: Stroke, Hyperhomocysteinemia, Orbitofrontal Syndrome.

\section{BACKGROUND/INTRODUCTION :}

Strokes in young are not rare and often pose a diagnostic challenge in young adults [1-2]. The proportions of strokes in young range between $19 \%$ and $30 \%$ in developing countries [3]. The types of strokes can be range between $21 \%$ and $78 \%$ for ischemic stroke, $3.7 \%$ to $38.5 \%$ for intracerebral haemorrhage and $9.6 \%$ to $55.4 \%$ for subarachnoid haemorrhage(2).One of the risk factors for ischemic stroke in young is Hyperhomocysteinemia wherein there a 2.5 -fold increased risk of stroke [4]. In patients with hyperacute strokes of right hemisphere a variety of cognitive syndromes can be seen [5]. Here we describe a case of stroke in young with Hyperhomocysteinemia presenting with orbitofrontal syndrome.

\section{CASE DESCRIPTION :}

Mr. X,37 years married male patient, residing with his nuclear family and was working as a bus driver. The patient had complaints of on and off chronic headache of more than 8 months duration, in back of the head (occipital region) relieved with over the counter (OTC) analgesics. Patient was brought to casualty with one day abrupt onset history of left sided weakness with multiple episodes of projectile vomiting and altered sensorium. On examination he was normotensive with Glasgow Coma Scale (GCS) score of E3V4M5 (12/15). His pupils were $2 \mathrm{~mm}$ and reactive, decreased movements in left upper and lower limbs with left extensor plantar response.
Intravenous (I.V) Mannitol $100 \mathrm{mg}$ stat dose was given and CT Brain was done which showed a large > 80\% Right Middle Cerebral Artery (MCA) territory infarct with significant mass effect and midline shift of $3 \mathrm{~mm}$ to left. Additionally, severe diffuse enema with hyperdense MCA and Anterior Cerebral Artery (ACA) arteries suggestive of acute thrombus were noted. Patient was admitted under neurosurgery department.

GCS of patient worsened rapidly from E3V4M6 to E1V2M5 and hence an emergency right fronto -temporo parietal decompressive craniectomy and lax duraplasty was performed. X-Ray showed evidence of aspiration pneumonia for which physicians' opinion was sought and started on antibiotics. On post-operative day 2 he showed no significant neurological improvement for which a repeat CT Scan was done which revealed evolution of infarct to involve entire Internal Carotid Artery (ICA) territory with minimal hemorrhagic transformation and increase in midline shift despite craniectomy status. Antienema measures were increased (started on hypertonicsaline/steroids along with mannitol). In view of possible need for prolonged airway support he was taken up for elective tracheostomy. With regular physiotherapy and nursing care patient's general condition and GCS improved to E4V1M6 and was weaned of ventilator. Further investigations for 'stroke-inyoung' revealed large right extracranial ICA erythematous plaque with $>75 \%$ luminal 
narrowing for which he was started on Antiplatelett medications. Serum homocysteine level was $53 \mu \mathrm{mol} / \mathrm{L}$ (normal<30) for which he was started on Folic acid/Vit B12/Vit B6 supplementation. He was diagnosed to have Hyper Homocystenemia with ICA territory malignant infarct with severe raised intra cranial pressure.

He was discharged with a GCS of E4M6V4, with stable vitals, on Tracheostomy tube on room air. He was accepting oral feeds and on Clean intermittent catheterization. He had left hemiplegia, comprehension, reading, writing and speech was grossly intact. Patient's family were educated about the diagnosis, need for continued physiotherapy, nursing, and tracheostomy care. He was discharged with levetiracetam 1000mg, amantadine $200 \mathrm{mg}$, tolperisone $150 \mathrm{mg}$, syrup glycerol 30ml three times a day, tolvaptan $15 \mathrm{mg}$, ecospirin $75 \mathrm{mg}$, vitamin supplements.

About 2 months later he was admitted and underwent right fronto-temporo-parietal cranioplasty using autologous bone graft titanium plate and screws alongside removal of tracheostomy (during which time aspirin was withheld transiently). Physician advice for investigating stroke in young with thromphophilia profile, anti-thrombin III, protein $\mathrm{S}$, protein $\mathrm{S}$, anti-phospholipid antibody levels along with homocysteine levels were followed up and advised long term anti-platelet therapy. Patient was restarted on aspirin and rest of the medications were continued to be rationalised accordingly on follow-up.

On follow -up patient presented with behavioural features for which he was referred to psychiatrist. On psychiatric evaluation, post -stroke 2 months history of unprovoked irritability with restlessness, distractibility, verbal abusiveness and repetitiveness, increased demands for food and hunger, decreased sleeping, altered bladder habits even after removal of catheter. In addition, patient had a history of alcohol and nicotine abuse but had been abstinent for 2 months. On physical examination of the patient post-stroke neurological deficits were evident. On mental status examination, rapport was difficult, patient was conscious, oriented, inattentive, with minimental status examination (MMSE) scoring at $25 / 30$, intact comprehension, increased reaction time, relevant and coherent speech with perseveration, dysphoric affect and was uncooperative for further detailed evaluation.
Patient received a diagnosis of Other mental disorders due to brain damage and dysfunction and to physical disease (Orbitofrontal Syndrome) [6]. Patient was started on night dose of Tablet Olanzapine $5 \mathrm{mg}$ and was asked to review on a monthly basis. In the subsequent 3 visits, Tablet Olanzapine was optimised to $15 \mathrm{mg}$ night dose with significant improvement in his behavioural disturbances. The need for a detailed neuropsychological assessment was explained to the family members.

\section{DISCUSSION :}

By 2050 about $80 \%$ of stroke events would occur in developing countries of the world [7]. Indian studies revealed that about $15 \%$ of strokes occur in people aged below 40 years of age [8]. In India, young age group can be restricted to 15-49 years as in this age group unique causes can be seen [9]. Ischemic type of stroke can have common cardioembolic causes like congenital heart diseases, rheumatic heart disease; vasculitis due to infections, antiphospholipid syndrome and less common causes like Hyperhomocysteinemia. Hemorrhagic causes of stroke include arteriovenous malformations, bleeding disorders and venous causes include oral contraceptives, connective tissue disease [8] [10]. Amongst these cerebral venous thrombosis and rheumatic heart disease are the leading causes whereas hyperhomocystenemia [11] being an uncommon cause; thus, making this case a notable one.

In this case an emergency neurosurgical intervention was warranted and reducing mortality as decompressive craniectomy improves survival in patients with malignant MCA infarction in comparison to conservative management [12]. Hyperhomocystenemia was treated with folic acid and vitamin supplementation [13]. In this case neuroimaging CT scan findings showed the ischemic stroke involving Right MCA, ICA territories which can result in orbitofrontal syndrome [14]. Some orbitofrontal syndrome features seen in this patient were behavioural disturbances, inattention and distractibility, verbal foul language disinhibition, hyperphagia, perseveration [15]. In view of patient having left hemiplegia with spasticity over duration of 2 months requiring physiotherapy, for his neuropsychiatric manifestation anti-psychotic olanzapine was used [16-17]. With subsidence in his inattention and behavioural disturbances, neuropsychological assessment of the patient 
would add data on such deficits amongst youngadult stroke patients [18]. Holistic team approach of early neurosurgical intervention, management of comorbid illness by Internal Medicine and ENT Physicians along with behavioural management by psychiatrist helped in reducing morbidity and preventing further decline in quality of life of the patient [19].

\section{REFERENCES :}

[1] Bevan, H., Sharma, K., \& Bradley, W. (1990). Stroke in young adults. Stroke, 21(3), 382-386.

[2] Incidence of Stroke in Young Adults: A Review. Available from: https://www.hindawi.com/journals/srt/2011/53567 2/

[3] Kittner, S. J., McCarter, R. J., Sherwin, R. W., Sloan, M. A., Stern, B. J., Johnson, C. J., ... \& Price, T. R. (1993). Black-white differences in stroke risk among young adults. Stroke, 24(12 Suppl), 113-115.

[4] Boushey, C. J., Beresford, S. A., Omenn, G. S., \& Motulsky, A. G. (1995). A quantitative assessment of plasma homocysteine as a risk factor for vascular disease: probable benefits of increasing folic acid intakes. Jama, 274(13), 1049-1057.

[5] Starkstein, S. E., \& Robinson, R. G. (1997). Mechanism of disinhibition after brain lesions. The Journal of nervous and mental disease, 185(2), 108-114.

[6] bluebook.pdf [Internet]. Available from: https://www.who.int/classifications/icd/en/bluebo ok.pdf

[7] Feigin, V. L. (2007). Stroke in developing countries: can the epidemic be stopped and outcomes improved?. The Lancet Neurology, 6(2), 94-97.

[8] Stroke in Young in India [Internet]. Available from:

https://www.hindawi.com/journals/srt/2011/36862 9/

[9] Prasad, K., \& Singhal, K. K. (2010). Stroke in young: An Indian perspective. Neurology India, 58(3), 343.

[10] Biswas, A., Ranjan, R., Meena, A., Akhter, M. S., Yadav, B. K., Munisamy, M., ... \& Saxena, R. (2009). Homocystine levels, polymorphisms and the risk of ischemic stroke in young Asian Indians. Journal of Stroke and Cerebrovascular Diseases, 18(2), 103-110.

[11] Dhamija, R. K., Gaba, P., Arora, S., Kaintura, A., Kumar, M., \& Bhattacharjee, J. (2009). Homocysteine and lipoprotein (a) correlation in ischemic stroke patients. Journal of the neurological sciences, 281(1-2), 64-68.

[12] Foerch, C., Lang, J. M., Krause, J., Raabe, A., Sitzer, M., Seifert, V., ... \& Kessler, K. R. (2004). Functional impairment, disability, and quality of life outcome after decompressive hemicraniectomy in malignant middle cerebral artery infarction. Journal of neurosurgery, 101(2), 248-254.

[13] The Treatment of Hyperhomocysteinemia | Annual Review of Medicine [Internet]. Available from:

https://www.annualreviews.org/doi/abs/10.1146/a nnurev.med.60.041807.123308

[14] Chow, T. W. (2000). Personality in frontal lobe disorders. Current psychiatry reports, 2(5), 446-451.

[15] Murad, A. (1999). Orbitofrontal syndrome in psychiatry. L'encephale, 25(6), 634-637.

[16] Shah, S. K. (1979). Disturbances of muscle tone in the paralysed upper extremity following hemiplegia. Australian Journal of Physiotherapy, 25(6), 243-249.

[17] Tarsy, D., Baldessarini, R. J., \& Tarazi, F. I. (2002). Effects of newer antipsychotics on extrapyramidal function. CNS drugs, 16(1), 2345.

[18] Cao, M., Ferrari, M., Patella, R., Marra, C., \&Rasura, M. (2007). Neuropsychological findings in young-adult stroke patients. Archives of Clinical Neuropsychology, 22(2), 133-142.

[19] Marini, C., Totaro, R., De Santis, F., Ciancarelli, I., Baldassarre, M., \&Carolei, A. (2001). Stroke in young adults in the communitybased L'Aquila registry: incidence and prognosis. Stroke, 32(1), 52-56.

$* * * * * * * * * * *$ 